\title{
EDITORIAL
}

\section{The CVC and CRBSI: don't use it and lose it!}

\author{
K. B. Laupland ${ }^{1 *}$ (D, D. Koulenti ${ }^{2,3}$ and C. Schwebel ${ }^{4,5}$
}

(c) 2017 Springer-Verlag GmbH Germany, part of Springer Nature and ESICM

In a recent article in Intensive Care Medicine, van der Kooi and colleagues report on the PROHIBIT trial aimed at preventing catheter-related bloodstream infections (CRBSI) in intensive care units (ICU) [1]. Following a baseline observation period, the investigators randomly allocated 14 ICUs to interventions that included a catheter insertion and care and hand hygiene improvement strategies. Participating ICUs were geographically distributed in Europe among 11 culturally diverse countries and were university-affiliated in one half of cases. Catheter insertion practices and hand hygiene adherence improved with the assigned interventions and these had significant reduction in the primary outcome of incidence density of CRBSI.

This study was a major undertaking that involved more than 35,000 central venous catheter (CVC) insertions among more than 25,000 patients admitted to ICUs. The study may be considered to have two key findings. First, the authors were able to successfully demonstrate that their interventions could improve hand hygiene adherence and catheter insertion and management techniques. Second, that these interventions resulted in a reduction in CRBSI. This study builds on and is a major contribution to the body of literature on CRBSI prevention in the ICU.

It is an important observation that the hand hygiene component of this study improved adherence to hand hygiene practices and that in turn this was associated with a reduction in CRBSI. Numerous previous studies have both demonstrated the importance of hand hygiene in reduction of nosocomial infections, and that interventions to improve adherence are associated with

\footnotetext{
*Correspondence: klaupland@gmail.com

${ }^{1}$ Intensive Care Unit, Royal Inland Hospital, 311 Columbia Street,

Kamloops, BC V2C 2T1, Canada

Full author information is available at the end of the article
}

improved outcomes [2-4]. However, much of the existing literature is based on "pre-post" intervention designs that have major methodological limitations. This study demonstrated the benefits of hand hygiene and catheter management on a reduction in CRBSI in a prospective controlled clinical trial.

Although not surprising based on contemporary observations elsewhere, it is disappointing that hygiene compliance at baseline averaged $49 \%$ in this study [5]. Hand hygiene is a relatively simple, inexpensive, and effective intervention that has few barriers to implementation [4]. However, while programmatic and infrastructural aspects may influence practices, the greatest challenges for successful hand hygiene adherence is human behavior and its resistance to change [6]. We contend that the rationale for and body of literature to support the benefit of hand hygiene is adequate. We must therefore shift our research efforts away from further attempts to demonstrate the effectiveness of hand hygiene toward a better understanding the determinants of hand hygiene adherence, knowledge translation, and implementation and maintenance of these practices [7-11].

As with hand hygiene, it is important to note that the catheter insertion and management intervention also led to a reduction in CRBSI incidence density [1]. Previous works have looked at this topic most commonly using the "pre-post" design with bundles of care [12]. The PROHIBIT study included numerous items on its catheter insertion and management protocol. The possibility exists that there may be additional aspects that could be added to this protocol that may further improve it such as use of ultrasound guidance or requirement for direct supervision of operators with limited experience. However, given the demonstrated benefit and that the $\mathrm{CVC}$

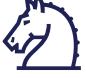 Springer}


insertion and management protocol prescribed in the PROHIBIT study is not onerous, we believe this represents a reasonable standard for implementation in ICUs.

While it is important to devise and implement means to reduce infectious complications of CVCs, it must be recognized that the only way to $100 \%$ ensure prevention of a CRBSI in a given patient is to avoid inserting a $\mathrm{CVC}$ in the first place. The overall utilization of CVCs decreased significantly in the PROHIBIT study from 88.3 to $73.5 \mathrm{CVC}$ days/ICU patient-days (Table S3), and this was likewise associated with a reduction in CRBSI [1].

Central venous catheters have many potential indications for use in ICU patients as listed in Table 1. While by virtue of the severity of their illness and need for complex care and monitoring, many or most patients admitted to ICUs will require a CVC. Indeed, it is our experience that many members of the ICU team view CVC insertion as a routine or standard procedure associated with the admission to ICU. However, like with any intervention that has the potential for harm, we must be judicious with the use of CVCs and recognize that minimizing their use is potentially an effective way to reduce CRBSI. Although there is a paucity of clinical trial data [13], an increasing body of observational literature is challenging dogma and questioning the necessity for routine CVC insertion for "classical" indications such as lower dose vasopressor infusion [14-17].

In summary, the PROHIBIT study is an important work and contribution to the critical care literature. It confirms the importance of hand hygiene and attention to $\mathrm{CVC}$ insertion procedures and management on reducing the incidence of CRBSI. Based on the results of this study and the vast previous literature, we believe that there is compelling evidence to support broad implementation of hand hygiene and catheter insertion and management protocols in our ICUs. However, further moving forward we must also address that the "root problem" of CRBSIs is the CVC itself. The onus is on us to use these devices judiciously in our clinical practices. Further

Table 1 Potential reasons for insertion of a central venous catheter among patients admitted to intensive care units

\begin{tabular}{l} 
Indication \\
Central venous pressure measurement \\
Venous blood gas measurement \\
Vasopressor and inotrope infusion \\
Volume resuscitation \\
Parenteral nutrition \\
Hemodialysis access \\
Ease of multiple venous access (i.e., multi-lumen catheter) \\
Introducer for transvenous pacemaker or pulmonary artery catheter \\
\hline
\end{tabular}

research efforts aimed at optimizing their safe non-use is warranted.

\section{Author details}

${ }^{1}$ Intensive Care Unit, Royal Inland Hospital, 311 Columbia Street, Kamloops, BC V2C 2T1, Canada. ${ }^{2}$ 2nd Critical Care Department, Attiko' University Hospital, Athens, Greece. ${ }^{3}$ Burns, Trauma and Critical Care Research Centre, UQ Centre for Clinical Research, Faculty of Medicine, The University of Queensland, Brisbane, Australia. ${ }^{4}$ Medical Intensive Care Unit, Grenoble University Hospital, Grenoble, France. ${ }^{5}$ Integrated Research Center, Inserm U1039, Radiopharmaceutical Bioclinical Mixed Research Unit, University of Grenoble-Alpes, Grenoble, France.

Compliance with ethical standards

Conflicts of interest

The authors have no conflicts of interest to disclose.

Received: 12 December 2017 Accepted: 15 December 2017

Published online: 26 December 2017

References

1. van der Kooi T, Sax H, Pittet D, van Dissel J, van Bentham B, Walder B, Cartier V, Clack L, de Greef S et al (2018) Prevention of hospital infections by intervention and training (PROHIBIT): results of a pan-European cluster-randomized multicenter study to reduce central venous catheter-related bloodstream infections. Intensive Care Med. https://doi. org/10.1007/s00134-017-5007-6

2. Liu S, Wang M, Wang G, Wu X, Guan W, Ren J (2017) Microbial characteristics of nosocomial infections and their association with the utilization of hand hygiene products: a hospital-wide analysis of 78,344 cases. Surg Infect (Larchmt) 18:676-683

3. Shabot MM, Chassin MR, France AC, Inurria J, Kendrick J, Schmaltz SP (2016) Using the Targeted Solutions Tool(R) to improve hand hygiene compliance is associated with decreased health care-associated infections. Jt Comm J Qual Patient Saf 42:6-17

4. Thi Anh Thu L, Thi Hong Thoa V, Thi Van Trang D, Phuc Tien N, Van Thuy D, Thi Kim Anh L, Wertheim HF, Truong Son N (2015) Cost-effectiveness of a hand hygiene program on health care-associated infections in intensive care patients at a tertiary care hospital in Vietnam. Am J Infect Control 43:e93-e99

5. Erasmus V, Daha TJ, Brug H, Richardus JH, Behrendt MD, Vos MC, van Beeck EF (2010) Systematic review of studies on compliance with hand hygiene guidelines in hospital care. Infect Control Hosp Epidemiol 31:283-294

6. Erasmus V, Brouwer W, van Beeck EF, Oenema A, Daha TJ, Richardus JH, Vos MC, Brug J (2009) A qualitative exploration of reasons for poor hand hygiene among hospital workers: lack of positive role models and of convincing evidence that hand hygiene prevents cross-infection. Infect Control Hosp Epidemiol 30:415-419

7. Battistella G, Berto G, Bazzo S (2017) Developing professional habits of hand hygiene in intensive care settings: an action-research intervention. Intensive Crit Care Nurs 38:53-59

8. King D, Vlaev I, Everett-Thomas R, Fitzpatrick M, Darzi A, Birnbach DJ (2016) "Priming" hand hygiene compliance in clinical environments. Health Psychol 35:96-101

9. Lydon S, Power M, McSharry J, Byrne M, Madden C, Squires JE, O'Connor P (2017) Interventions to improve hand hygiene compliance in the ICU: a systematic review. Crit Care Med 45:e1165-e1172

10. Musu M, Finco G, Mura P, Landoni G, Piazza MF, Messina M, Tidore M, Mucci M, Campagna M, Galletta M (2017) Controlling catheter-related bloodstream infections through a multi-centre educational programme for intensive care units. J Hosp Infect 97:275-281

11. Diegel-Vacek L, Ryan C (2016) Promoting hand hygiene with a lighting prompt. HERD 10:65-75 
12. Ista E, van der Hoven B, Kornelisse RF, van der Starre C, Vos MC, Boersma E, Helder OK (2016) Effectiveness of insertion and maintenance bundles to prevent central-line-associated bloodstream infections in critically ill patients of all ages: a systematic review and meta-analysis. Lancet Infect Dis 16:724-734

13. Ricard JD, Salomon L, Boyer A, Thiery G, Meybeck A, Roy C, Pasquet B, Le Miere E, Dreyfuss D (2013) Central or peripheral catheters for initial venous access of ICU patients: a randomized controlled trial. Crit Care Med 41:2108-2115

14. Datar S, Gutierrez E, Schertz A, Vachharajani V (2017) Safety of phenylephrine infusion through peripheral intravenous catheter in the neurological intensive care unit. J Intensive Care Med. https://doi. org/10.1177/0885066617712214
15. Brewer JM, Puskarich MA, Jones AE (2015) Can vasopressors safely be administered through peripheral intravenous catheters compared with central venous catheters? Ann Emerg Med 66:629-631

16. Loubani OM, Green RS (2015) A systematic review of extravasation and local tissue injury from administration of vasopressors through peripheral intravenous catheters and central venous catheters. J Crit Care 30:653. e9-653.e17

17. Delgado T, Wolfe B, Davis G, Ansari S (2016) Safety of peripheral administration of phenylephrine in a neurologic intensive care unit: a pilot study. J Crit Care 34:107-110 